\title{
Evidence for Interlayer Band Shifts upon Lithium Intercalation in Graphite from Inelastic X-Ray Scattering
}

\author{
W. Schülke, A. Berthold, and A. Kaprolat \\ Institute of Physics, University of Dortmund, D-4600 Dortmund 50, Federal Republic of Germany
}

and

H.-J. Güntherodt

Institute of Physics, University of Basel, Ch-4056 Basel, Switzerland

(Received 1 February 1988)

\begin{abstract}
The dielectric function $\epsilon(\mathbf{q}, \omega)$ of Li-intercalated graphite $\left(\mathrm{LiC}_{6}\right)$ with $\mathbf{q} \| c$ axis was deduced from in elastic $\mathrm{x}$-ray scattering data and compared with $\epsilon(\mathbf{q}, \omega)$ of pristine graphite. A displacement and broadening of the first peak of $\operatorname{Im} \epsilon(\mathbf{q}, \omega)$ upon intercalation was found, which we attribute to a 2-eV shift of the "metal" $\sigma$ state of $\mathrm{LiC}_{6}$ to lower energies relative to the $\sigma$-type interlayer state of graphite.
\end{abstract}

PACS numbers: 71.25.Pi, 71.20.Cf, 78.70.Ck

First-stage Li-intercalated graphite $\left(\mathrm{LiC}_{6}\right)$ is one of the alkali-metal-graphite intercalation compounds whose electronic structure has been thoroughly investigated both experimentally ${ }^{1-6}$ and theoretically. ${ }^{7-10}$ It is commonly accepted that, upon intercalation of $\mathrm{Li}$, the electrons are completely transferred from the $\mathrm{Li} 2 s$ states to previously empty carbon $\pi$ states. This charge transfer can be understood within the framework of the so-called rigid-band model, where the in-plane part of the $\mathrm{LiC}_{6}$ band structure is the result of backfolding of the $2 \mathrm{D}$ bands of graphite into the smaller Brillouin zone of $\mathrm{LiC}_{6}$. Nevertheless, the full understanding of the electron structure of alkali-metal-graphite intercalating compounds also needs consideration of changes of the band structure beyond the rigid-band model, both due to the existence of $\mathrm{Li}$ ions between the graphite layers and as a result of the altered stacking of graphite layers. The so-called interlayer states ${ }^{8}$ of graphite are important candidates for those changes since strong hybridization with $\mathrm{Li} 2 s$ states is rather likely. Angle-resolved inverse-photoemission (ARIPE) results ${ }^{4}$ on $\mathrm{LiC}_{6}$ and graphite have corroborated this assumption.

It is the aim of this Letter to present further evidence for nonrigid behavior of the $\mathrm{LiC}_{6}$ conduction bands by comparison of inelastic $\mathrm{x}$-ray-scattering spectroscopy (IXSS) data of $\mathrm{LiC}_{6}$ with those of pristine graphite. In contrast to electron-energy-loss spectroscopy, ${ }^{5}$ measurements with $\mathbf{q} \| c$ axis could be performed with IXSS, so that a new spectroscopic access to the interlayer states was opened via dipole-allowed transitions from $\pi$ states to the interlayer states.

IXSS measures the dynamic structure factor $S(\mathbf{q}, \omega)$ and the imaginary part of the inverse dielectric function, $\operatorname{Im} \epsilon^{-1}(\mathbf{q}, \omega)$, both on an absolute scale, by utilization of the oscillator strength sum rule, ${ }^{11}$ so that $\epsilon_{1}(\mathbf{q}, \omega)$ $\equiv \operatorname{Re} \epsilon(\mathbf{q}, \omega)$ and $\epsilon_{2}(\mathbf{q}, \omega) \equiv \operatorname{Im} \epsilon(\mathbf{q}, \omega)$ can be obtained by means of Kramers-Kronig transformation. It is the well-known relation between $\operatorname{Im} \epsilon(\mathbf{q}, \omega)$ and symmetry projection of the joint density of states (DOS) that brings about the desired band-structure information.

The IXSS measurements of $S(\mathbf{q}, \omega)$ with $0.8-\mathrm{eV}$ resolution were performed by use of synchrotron radiation from the DORIS storage ring at DESY, monochromatized to an energy of $8 \mathrm{keV}$ by a $\mathrm{Ge}(311)$ double-crystal setting. The scattered radiation was analyzed by means of a spherically bent $\mathrm{Si}(444)$ crystal. Details of the experimental setup and of data processing are described elsewhere. ${ }^{11,12}$ The graphite sample was a highly oriented pyrolytic graphite (HOPG) disk of 1.3-mm thickness. The $\mathrm{LiC}_{6}$ sample of $2-\mathrm{mm}$ thickness and $20 \times 30-\mathrm{mm}^{2}$ lateral dimension was obtained by a liquid-phase reaction under clean Ar atmosphere with HOPG. Stage and homogeneity of the $\mathrm{LiC}_{6}$ sample was controlled by energy-dispersive $\mathrm{x}$-ray diffraction both before and after the experiment. The $\mathrm{LiC}_{6}$ sample was transferred from argon-filled glass ampules to the scattering chamber via an argon ambient glove bag and then held at $10^{-6}$ Torr. The bright golden surface did not deteriorate during the six days of the experiment.

The sequence of presentation of the results is as follows: In the first place, carbon $1 s$ core-excitation spectra (CES) will be presented, displayed together with angleresolved $\mathrm{x}$-ray emission spectroscopy (ARXES) results in order to fix the Fermi level and to demonstrate the nearly rigid-band behavior of the involved conduction bands. In the second place, the strong changes of the valence-electron excitation spectra with $\mathrm{q} \| c$ will be discussed in terms of hybridization of the interlayer states with states originating from $\mathrm{Li} 2 s$.

In Fig. 1 the carbon $1 s$ CES's of $\mathrm{LiC}_{6}$ and HOPG are compared. Within the limits of a $2 \mathrm{D}$ representation of the band structure, and with utilization of dipole selection rules, the $\mathrm{q} \| c$-axis spectra and the $\mathrm{q} \perp c$-axis spectra reflect a symmetry-projected partial DOS of $\pi^{*}$ states 


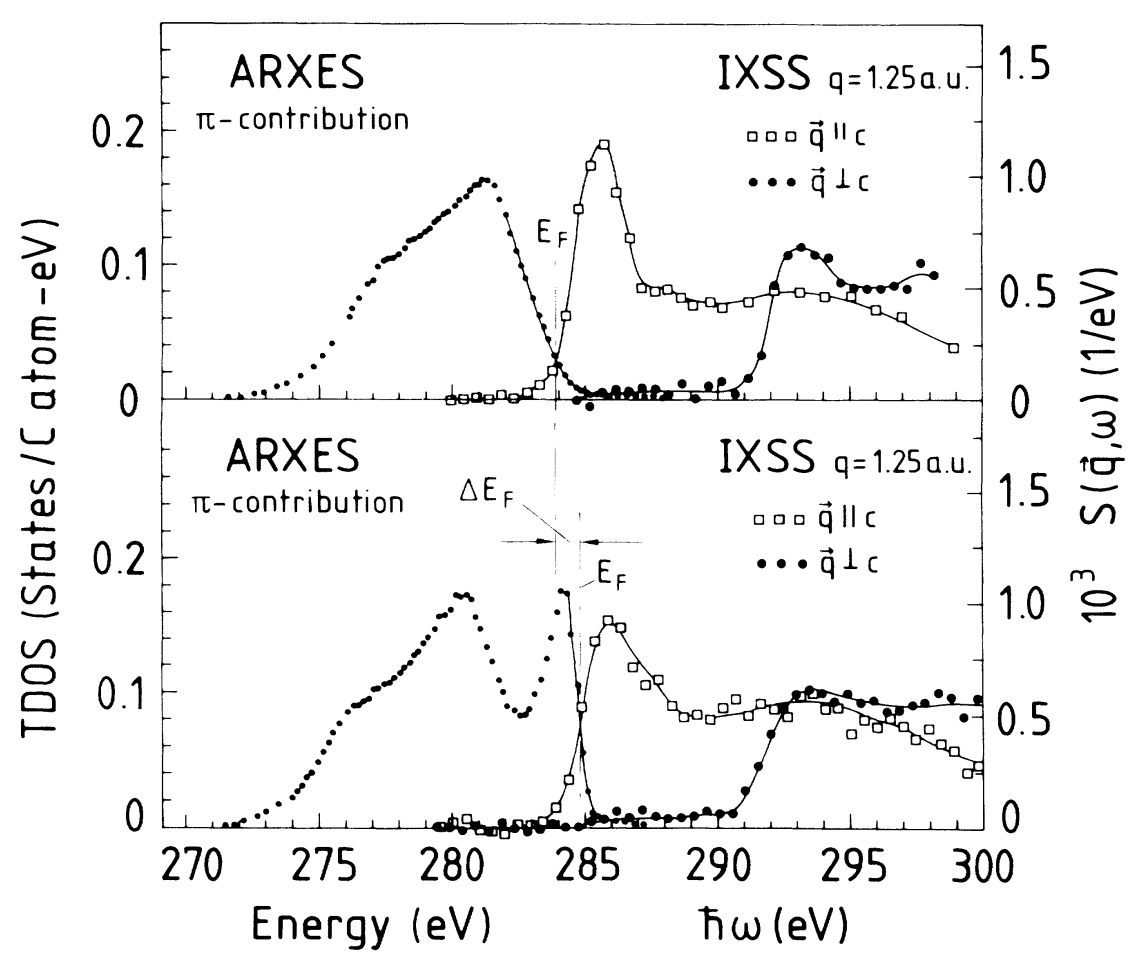

FIG. 1. Right-hand part, right-hand scale: C $1 s$ core contribution to $S(\mathbf{q}, \omega)$ of HOPG (upper part) and $\mathrm{LiC}_{6}$ (lower part), with $\mathbf{q} \| c$ axis (open squares) and $\mathbf{q} \perp c$ axis (filled circles). Left-hand part, left-hand scale: $\pi$ contribution of the $C K \times$-ray emission band for graphite (upper part) and $\mathrm{LiC}_{6}$ (lower part), according to Ref. 6. $E_{\mathrm{F}}$, estimated position of the Fermi level; $\Delta E_{\mathrm{F}}$, Fermilevel shift.

and $\sigma^{*}$ states, respectively. ${ }^{12}$ Together with the IXSS data the corresponding $\pi$ contribution to the ARXES spectra ${ }^{6}$ is displayed. The intersection of ARXES spectra with the IXSS spectra indicates the Fermi energy $E_{\mathrm{F}}$ relative to $\mathrm{C} 1 s$.

Hence a shift of $E_{\mathrm{F}}$ to higher energies by $\Delta E_{\mathrm{F}}$ $=1.0 \pm 0.3 \mathrm{eV}$ follows upon $\mathrm{Li}$ intercalation, if we ascribe the measured ${ }^{2}$ lowering of the $\mathrm{C} 1 s$ binding energy, $\Delta E_{B}=0.8 \mathrm{eV}$, solely to a change of the core-hole relaxation. Otherwise, an unknown fraction $p$ of $\Delta E_{B}$ had to be added to $\Delta E_{\mathrm{F}}$. We attribute the first pronounced peak in the $\mathbf{q} \| c$ CES to regions of high DOS of unoccupied $\pi$ states in graphite $\left(\pi_{2}\right.$ in Fig. 2 near $\left.M\right)$ and $\pi$ originated states in $\mathrm{LiC}_{6}$, respectively. We explain the reduction of peak height and a small shift $(\approx 0.5 \mathrm{eV})$ of the peak position to higher energies (contrary findings in electron-energy-loss spectroscopy ${ }^{5}$ must be left unexplained) as a result of the filling of part of the graphite $\pi$ states by electrons transferred from $\mathrm{Li} 2 s$ states. Hence our results are consistent with a nearly rigid-band behavior of graphite $\pi$ states upon intercalation. We interpret the steep rise of the $\mathbf{q} \perp c$ CES as brought about by the onset of the contribution to the DOS of unoccupied graphite $\sigma$ states $\left(\sigma_{5}\right.$ and $\sigma_{6}$ in Fig. 2) and $\mathrm{LiC}_{6} \sigma-$ derived states, respectively, with appropriate symmetry. ${ }^{11}$ The inflection points of this rise are exactly at the same position for HOPG and $\mathrm{LiC}_{6}$, so that this $\sigma$-DOS feature has not been shifted by more than $p \Delta E_{B}$ upon Li intercalation, an indication of a nearly rigid-band behavior of those graphite $\sigma$ states that have a strong overlap with $\mathrm{C} 1 s$ states. The $\sigma$-type interlayer states $\sigma_{4}$ in Fig. 2 do not contribute appreciably to the C $1 s$ CES, since their overlap with $\mathrm{C} 1 s$ can be neglected. The vanishing

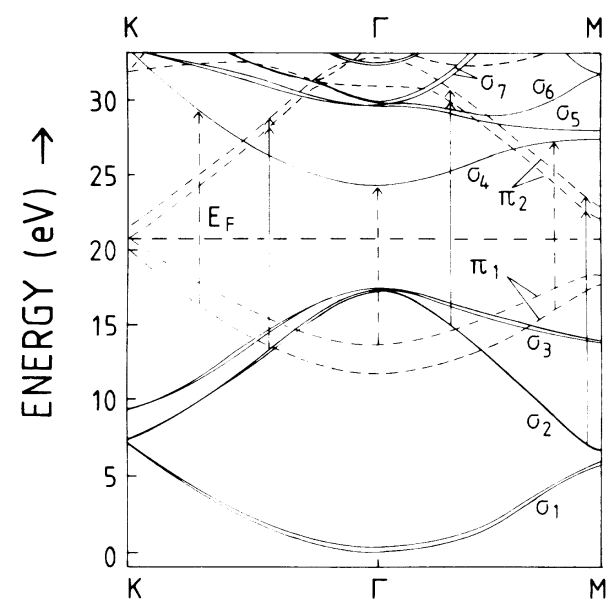

FIG. 2. Partial energy-band structure of graphite according to Ref. 13. Solid curves: $\sigma$ bands; dashed curves: $\pi$ bands. The arrows indicate some dipole-allowed vertical transitions for $\mathbf{q} \| c$ axis. 
of the peak at $293 \mathrm{eV}$ in the $\mathbf{q} \perp c$ spectrum of graphite upon intercalation can be interpreted as due to $\sigma$-band folding.

The normalized $S(\mathbf{q}, \omega)$ spectra of HOPG and $\mathrm{LiC}_{6}$ with $\mathbf{q} \| c$ axis $(q=0.59$ a.u. $)$ are presented in Fig. 3(a); the real $\left(\epsilon_{1}\right)$ and imaginary $\left(\epsilon_{2}\right)$ parts of $\epsilon(\mathbf{q}, \omega)$ are presented in Fig. 3(b). The main feature in the $\epsilon_{2}$ spectra of graphite is the strong peak at $14.6 \pm 0.2 \mathrm{eV}$ which is shifted by $2.0 \pm 0.4 \mathrm{eV}$ to $12.6 \pm 0.2 \mathrm{eV}$ upon $\mathrm{Li}$ intercalation. The FWHM of this peak increases from $4.5 \pm 0.2 \mathrm{eV}$ in $\mathrm{HOPG}$ to $6.0 \pm 0.2 \mathrm{eV}$ in $\mathrm{LiC}_{6}$. In discussion of these findings in terms of the band structure, all transitions can be considered as vertical, since $q \approx 2 \bar{\Gamma} \bar{A}$.

On the basis of dipole selection rules it was deduced in Ref. 12 that the first peak of $\epsilon_{2 \|}$ in graphite is composed of two contributions which are only slightly separated in energy so that they could not be resolved experimentally. The one peak contribution at higher energy transfer $(\approx 16.5 \mathrm{eV})$ is due to vertical transitions between the $\sigma_{2}$ band and the nearly parallel $\pi_{2}$ band (solid arrow in Fig. 2). The second contribution at lower energy transfer $(\simeq 13.5 \mathrm{eV})$ originates from vertical transitions (dashed arrow in Fig. 2) between the $\pi_{1}$ band and the nearly parallel $\sigma$-type interlayer band ( $\sigma_{4}$ in Fig. 2 ). This tran- sition contributes to $\epsilon_{2 \|}$ because of the considerable extension of the $\pi$ states into the interlayer region. A double-peak structure of the above type was also predicted in Ref. 10.

Hence the shift and broadening of the first $\epsilon_{2 \|}$ peak upon intercalation can be explained as follows: The energy of the $\sigma_{2} \rightarrow \pi_{2}$ transition remains roughly the same because of the nearly rigid-band behavior both of the $\sigma_{2}$ band (see Ref. 1) and of the $\pi_{2}$ band (see our CES results). In contrast, the energy of the $\pi_{1} \rightarrow \sigma$-type interlayer state $\left(\sigma_{4}\right)$ transition is shifted by at least $2 \mathrm{eV}$ to lower values, since the "metal" $\sigma$ band of $\mathrm{LiC}_{6}$ (notation according to Ref. 10) is by $2 \mathrm{eV}$ lower in energy than the $\sigma$-type interlayer state of pristine graphite, from which it originates, possible because of strong hybridization with the $\mathrm{Li}$ metal $2 s$ band, ${ }^{9}$ whereas the $\pi_{1}$ band behaves nearly rigidly (see Ref. 1). In terms of the hybrid point of view, the measured energy shift of at least $2 \mathrm{eV}$ must be considered as a measure of the degree of hybridization. Fauster et al. ${ }^{4}$ have found in ARIPE the corresponding energy shift to be $1.4 \mathrm{eV}$; calculations of Holzwarth, Louie, and Rabii ${ }^{7}$ have determined the shift at $\Gamma$ to be $1.6 \mathrm{eV}$, resulting in a shift of $\approx 2 \mathrm{eV}$ of corresponding peaks in $\epsilon_{2 \|}$ (Ref.10) in excellent agreement with our measurements.

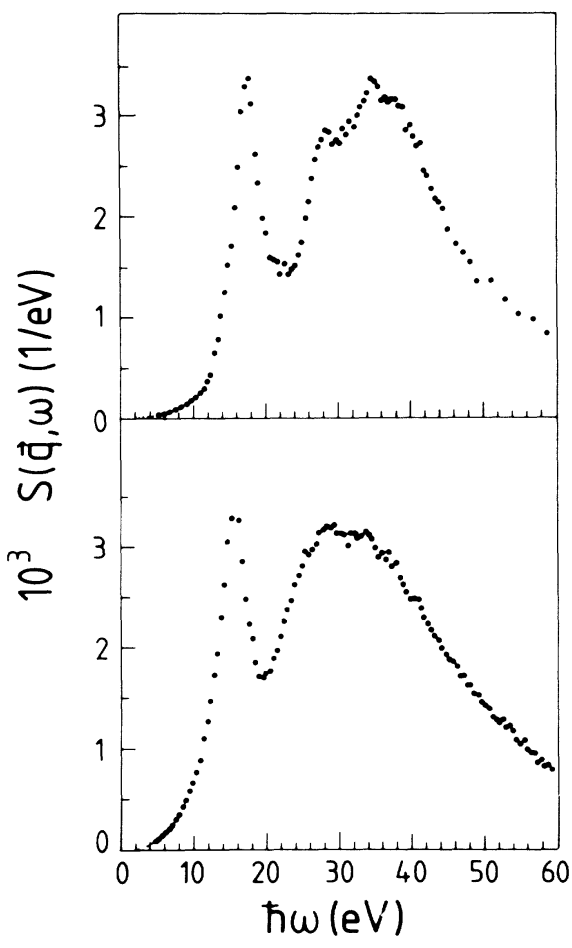

(a)

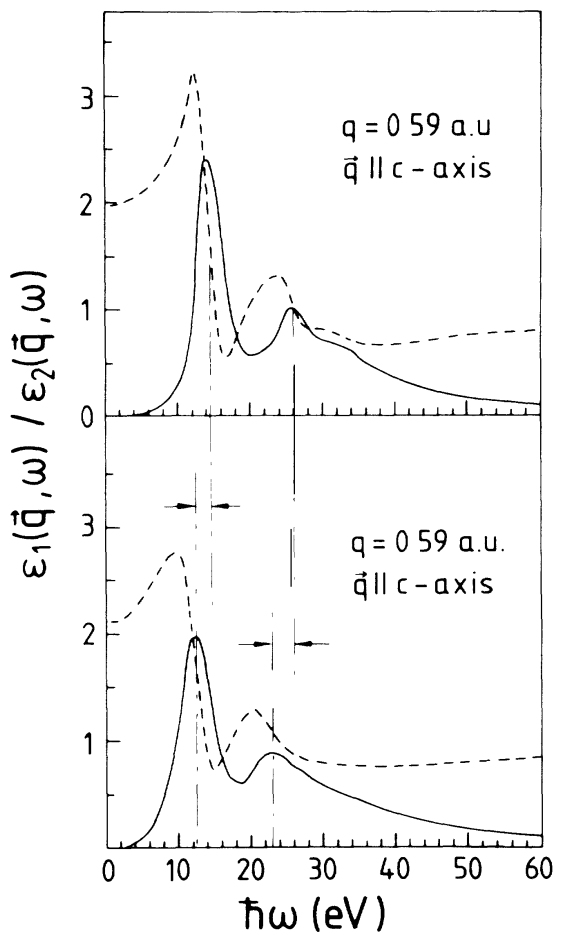

(b)

FIG. 3. (a) IXSS-measured $S(\mathbf{q}, \omega)$ for $\mathbf{q} \| c$ axis $\left(q=0.59\right.$ a.u.) of HOPG (upper part) and $\mathrm{LiC}_{6}$ (lower part). Small dots at low energy transfer indicate the less-reliable part of the spectrum because of heavy superposition by the Rayleigh line. (b) $\epsilon_{1}(\mathbf{q}, \omega)$ (dashed curve) and $\epsilon_{2}(\mathbf{q}, \omega)$ (solid curve) of HOPG (upper part) and $\mathrm{LiC}_{6}$ (lower part). Peak shifts upon intercalation are indicated. 
The shift by $3 \mathrm{eV}$ of the second peak in the $\epsilon_{2 \|}$ spectrum to lower energies upon intercalation could be explained as due to a shift to lower energies upon intercalation of the $\sigma$-type conduction bands with strong $k_{z}$ dispersion, which were found by Tatar and $\mathrm{Rabii}^{14}$ in their band calculations of graphite to be parallel to the "rigid" $\pi_{1}$ bands. ${ }^{12}$

In conclusion, we have directly measured for the first time the dielectric function $\epsilon(\mathbf{q}, \omega)$ with $\mathbf{q} \|_{c}$ axis of graphite and $\mathrm{LiC}_{6}$. Thus we could make accessible by dipole transition rules transitions from $\pi$ states to $\sigma$-type interlayer states utilizing their considerable spatial overlap. We could establish the energy shift of the interlayer states of graphite upon intercalation in agreement with theoretical predictions and ARIPE results.

We thank U. Bonse, K. Fischer, R. Nusshardt, and A. Kirfel for valuable help with the measurements at DORIS/HASYLAB (Hamburger Synchrotronstrahlungslabor) and V. Geiser for the $\mathrm{LiC}_{6}$ sample preparation. This work has been funded by the German Federal Ministry of Research and Technology (BMFT) under Contract No. 053347 XB 2. The financial support from the Swiss National Science Foundation for the preparation of the intercalation sample is acknowledged.
${ }^{1}$ W. Eberhardt, I. T. McGovern, E. W. Plummer, and J. E. Fischer, Phys. Rev. Lett. 44, 200 (1980).

${ }^{2}$ G. K. Wertheim, P. M. Th. M. Van Attekum, and S. Basu, Solid State Commun. 33, 1127 (1980).

${ }^{3}$ U. M. Gubler, P. Oelhafen, and H. J. Güntherodt, Solid State Commun. 44, 1621 (1982).

${ }^{4}$ Th. Fauster, F. J. Himpsel, J. E. Fischer, and E. W. Plummer, Phys. Rev. Lett. 51, 430 (1983).

${ }^{5}$ L. A. Grunes, I. P. Gates, J. J. Ritsko, E. J. Mele, D. P. Vincenzo, M. E. Preil, and J. E. Fischer, Phys. Rev. B 28, 6681 (1983).

${ }^{6}$ A. Mansour, S. E. Schnatterly, and J. J. Ritsko, Phys. Rev. Lett. 58, 614 (1987).

${ }^{7}$ N. A. Holzwarth, S. G. Louie, and S. Rabii, Phys. Rev. B 28, 1013 (1983).

${ }^{8}$ M. Posternak, A. Baldereschi, A. J. Freeman, E. Wimmer, and M. Weinert, Phys. Rev. Lett. 50, 761 (1983).

${ }^{9}$ N. A. W. Holzwarth, S. G. Louie, and S. Rabii, Phys. Rev. B 30, 2219 (1984).

${ }^{10}$ N. X. Chen and S. Rabii, Phys. Rev. B 31, 4784 (1985).

${ }^{11}$ W. Schülke, H. Nagasawa, S. Mourikis, and P. Lanzki, Phys. Rev. B 33, 6744 (1986).

${ }^{12}$ W. Schülke, U. Bonse, H. Nagasawa, A. Kaprolat, and A. Berthold, Phys. Rev. B (to be published).

${ }^{13}$ N. A. W. Holzwarth, S. G. Louie, and S. Rabii, Phys. Rev. B 26, 5382 (1982).

${ }^{14}$ R. C. Tatar and S. Rabii, Phys. Rev. B 25, 4126 (1982). 